\title{
METRIC SPACES WITH NICE CLOSED BALLS AND DISTANCE FUNCTIONS FOR CLOSED SETS
}

\author{
GERALD BeER
}

\begin{abstract}
A metric space $(X, d)$ is said to have nice closed balls if each closed ball in $X$ is either compact or the entire space. This class of spaces includes the metric spaces in which closed and bounded sets are compact and those for which the distance function is the zero-one metric. We show that these are the spaces in which the relation $F=\operatorname{Lim} F_{n}$ for sequences of closed sets is equivalent to the pointwise convergence of $\left\langle d\left(\cdot, F_{n}\right)\right\rangle$ to $d(\cdot, F)$. We also reconcile these modes of convergence with three other closely related ones.
\end{abstract}

\section{Introduction}

Let $C L(X)$ be the collection of closed nonempty subsets of a metric space $\langle X, d\rangle$. If $F_{1} \in C L(X)$ and $F_{2} \in C L(X)$, the Hausdorff distance between $F_{1}$ and $F_{2}$ is given by $h_{d}\left(F_{1}, F_{2}\right)=\sup \left(\left\{d\left(x, F_{1}\right)\right.\right.$ : $\left.\left.x \in F_{2}\right\} \cup\left\{d\left(x, F_{2}\right): x \in F_{1}\right\}\right)$. Distance so defined determines an infinite valued metric on $C L(X)$. Basic facts about Hausdorff distance can be found in Aubin [2], Castaing and valadier [6], or Klein and Thompson [12]. Most fundamentally $[2]$, the map $F \rightarrow d(\cdot, F)$ is an isometry of $\left(C L(X), h_{d}\right\rangle$

Received 4 March 1986.

Copyright Clearance Centre, Inc. Serial-fee code: 0004-9727/87 $\$ \mathrm{~A} 2.00+0.00$. 
into the space of real valued continuous functions on $X$ equipped with the infinite valued metric $\rho(f, g)=\sup \{|f(x)-g(x)|: x \in X\}$. Thus, a sequence $\left(E_{n}\right)$ in $C L(X)$ is $h_{d}$-convergent to a nonempty closed set $F$ if and only if $\left(d\left(\cdot, F_{n}\right)\right\rangle$ converges uniformly to $d(\cdot, F)$. As a result, pointwise convergence of $\left\langle d\left(\cdot, F_{n}\right)\right\rangle$ to $d(\cdot, F)$ is a weaker form of convergence than Hausdorff metric convergence of $\left\langle F_{n}\right\rangle$ to $F$. Recently, Lechicki and Levi [14] have shown that if $X$ has a countable dense subset $\left\{x_{n}: n \in z^{+}\right\} \quad$ then pointwise convergence of distance functions is convergence with respect to this metric on $C L(X)$ :

$$
\rho^{*}(A, B)=\sum 2^{-n} \cdot \frac{\left|d\left(x_{n}, A\right)-d\left(x_{n}, B\right)\right|}{1+\left|d\left(x_{n}, A\right)-d\left(x_{n}, B\right)\right|}
$$

In [3] the author made a feeble attempt to determine in which spaces pointwise convergence of distance functions for closed sets is equivalent to a standard notion of convergence of sets weaker than $h_{d}$-convergence. If $\left\langle F_{n}\right\rangle$ is a sequence in $C L(X)$, let $L i F_{n}$ (respectively $L s F_{n}$ ) be the set of points $y$ in $X$ each neighbourhood of which meets all but finitely (respectively infinitely) many sets $F_{n}$. If $L i F_{n}=L s F_{n}=F$, we write $F=\operatorname{Lim} F_{n}$ [13]. It is well known (see [2], [3] or [9]) that if $F \in C L(X)$ and $\left\langle F_{n}\right\rangle$ is a sequence in $C L(X)$ whose distance functions converge pointwise to the distance function of $F$, then $F=\operatorname{Lim} F_{n}$. Those spaces $X$ for which the converse holds include the spaces in which closed and bounded sets are compact, and are characterized as follows: whenever $\left\langle x_{n}\right\rangle$ is a sequence in $X$ with no cluster point, then for each $p$ and $x$ in $X$, we have $d(p, x) \leq \lim$ inf $d\left(p, x_{n}\right)$. It is one purpose of this paper to present more tangible descriptions of such spaces. More importantly, we study the relationship between convergence of distance functions for closed sets and convergence of sequences of sets with respect to two vietoris-type topologies on $C L(X)$, as well as the $h_{d}$-topology on $C L(X)$.

Keeping notation uncumbersome when discussing such matters is a nontrivial task. Motivated by standard notation for multifunctions [5], 
if $A \subset X$ we write $A^{+}$for $\{F \in C L(X): E \subset A\}$ and $A^{-}$for $\{F \in C L(X): \quad F \cap A \neq \emptyset\}$. A well-studied topology on $C L(X)$, which is consistent with pointwise convergence of distance functions when $X$ is ordinary Euclidean space, is the topology of closed convergence ${ }^{\tau} C[12]$, also called the Fell topology [8], generated by all sets of the form $\left(K^{c}\right)^{+}$with $K$ compact, and $V^{-}$with $V$ open. If $X$ is locally compact and separable, then ${ }^{\tau_{C}}$ is metrizable and topologizes the relation $F=\operatorname{Lim} F_{n}$ for sequences of sets (see [10] or [12]). If $X$ is locally compact, but not necessarily separable, it is still the case that ${ }^{2} C$ topologizes the convergence of nets of closed sets (with $F=\operatorname{Lim} F_{n}$ defined in the obvious way), in the sense that the convergence structure satisfies the net axioms of Kelley [11] (see for example, [16] or [12]). A natural misconception has arisen from this theory, which we would like to correct: local compactness of $X$ is necessary for the equivalence of the relation $F=\operatorname{Lim} F_{n}$ for sequences of closed sets and $\tau_{C}$-convergence of $\left\langle F_{n}\right\rangle$ to $F$. This is utterly false, a point that has been informally observed in [9].

LEMMA 1.0. Let $(X, d\rangle$ be a metric space, and let $F, F_{1}, F_{2}, \ldots$ be nonempty closed subsets of $X$. Then $F=\operatorname{Lim} F_{n}$ if and only if $\left\langle F_{n}\right\rangle$

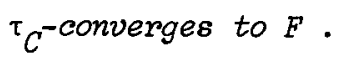

Proof. The relation $F=\operatorname{Lim} F_{n}$ means (i) $F \subset L i F_{n}$, and (ii) Ls $F_{n} \subset F$. By (i), if $F$ meets an open set $V$, then $\left\langle F_{n}\right\rangle$ meets $V$ eventually. By (ii), if $F$ misses a compact set $K$, then $\left\langle F_{n}\right\rangle$ misses $K$ eventually. Thus, $\left\langle F_{n}\right\rangle$ is ${ }^{\tau} C^{\text {-convergent to } F}$. Conversely, if $\left(F_{n}\right\rangle$ is ${ }^{\top} C$-convergent to $F$, we show (i) and (ii) hold. Condition (i) is immediate, for if $x \in F$ and $\varepsilon$ is positive, then ' $F$ ' must be in $\{w: d(x, w)<\varepsilon\}^{-}$eventually. Suppose (ii) fails. We can then find an increasing sequence $\left\langle n_{k}\right\rangle$ of positive integers and a sequence $\left\langle x_{k}\right\rangle$ in $X$ convergent to a point $p$ in $F^{c}$ such that for each $k, x_{k} \in F_{n_{k}}$. Since $F$ is closed, without loss of generality, we may assume each $x_{k}$ lies 
in $F^{f}$. As a result, $\{p\} \cup\left\{x_{k}: k \in Z^{+}\right\}$is a compact set that misses $F$, but which meets $\left\langle F_{n}\right\rangle$ frequently. We conclude that $\left\langle F_{n}\right\rangle$ does not $\tau_{C}$-converge to $F$.

Another topology of somewhat greater strength which would appear to be a prime candidate for one compatible with pointwise convergence of distance functions is generated by all sets of the form $\left(B^{C}\right)^{+}$with $B$ a closed ball in $X$ and $S^{-}$with $S$ an open ball in $X$. We call this topology the ball topology on $C L(X)$, and denote it by $\tau_{B}$ in the sequel. of course, "open ball" could be replaced by "open set" in the description of its subbase without enlarging the topology. The ball topology has been of some interest to Salinetti and wets in their ongoing study of weak convergence of probability measures [18]. Now if $F \in C L(X)$ and $K$ is a nonempty compact set for which $F \in\left(K^{c}\right)^{+}$, then there exists a finite collection of closed balls $B_{1}, \ldots, B_{n}$ in $X$ with $F \cap\left({\stackrel{n}{j}{ }_{1}}^{n} B_{j}\right)=\emptyset$ and $K \subset \underset{j=1}{n} B_{j}$. As a result $\tau_{C} \subset \tau_{B}$.

In the sequel $S_{\varepsilon}[p]$ (respectively $B_{\varepsilon}[p]$ ) will denote the open (respectively closed) ball of radius $\varepsilon$ with centre $p$ in $X$. Also, if $A \subset X$, we write $S_{\varepsilon}[A]$ for $\cup\left\{S_{\varepsilon}[x]: x \in A\right\}$, and call $S_{\varepsilon}[A]$ the E-parallel body of $A$. Of course, Hausdorff distance can be expressed in terms of parallel bodies:

$$
h_{d}\left(F_{1}, F_{2}\right)=\inf \left\{\varepsilon: S_{\varepsilon}\left[F_{1}\right] \supset F_{2} \text { and } S_{\varepsilon}\left[F_{2}\right] \supset F_{1}\right\}
$$

\section{The Main Results}

LEMMA 2.0. Let $F, F_{1}, F_{2}, \ldots$ be nonempty closed sets in a metric space $\langle X, d\rangle$. If $\left\langle E_{n}\right\rangle \tau_{B}$-converges to $F$, then $\left\langle d\left(\cdot, F_{n}\right\rangle\right\rangle$ converges pointwise to $d(\cdot, F)$. If $\left\langle d\left(\cdot, F_{n}\right)\right\rangle$ converges pointwise to $d(\cdot, F)$, then $\left\langle F_{n}\right\rangle{ }^{\top} C^{\text {-converges to } F}$ (see Proposition 2.3 of [9]).

Proof. Suppose $\left\langle F_{n}\right\rangle$ converges to $F$ in the ball topology. If $x \in F$ and $\varepsilon$ is positive, then $\left\langle F_{n}\right\rangle$ eventually lies in $\left(S_{\varepsilon}[x]\right)^{-}$. 
This means that for all $n$ sufficiently large, $F_{n}$ meets $S_{\varepsilon}[x]$. As a result, $d(\cdot, F) \geq \lim \sup d\left(\cdot, F_{n}\right)$. Suppose that $d(\cdot, F) \cdot \leq \lim \inf d\left(\cdot, F_{n}\right)$ fails. Then for some $p \in X$ and $\varepsilon>0$ there exists a subsequence $\left\langle F_{n_{k}}\right\rangle$ of $\left\langle F_{n}\right\rangle$ such that for each $k, d\left(p, F_{n_{k}}{ }^{\prime}<d(p, F)-\varepsilon\right.$. Let $\alpha=d(p, F)-\varepsilon ;$ we have $F \in\left[\left(B_{\alpha}[p]\right)^{c}\right]^{+}$, but for each $k$, $F_{n_{k}} \notin\left[\left(B_{\alpha}[p]\right)^{C}\right]^{+}$. Thus, $\left\langle F_{n}\right\rangle$ does not $\tau_{B}$-converge to $F$, a contradiction. We conclude that $\left\langle d\left(\cdot, F_{n}\right)\right\rangle$ converges pointwise to $d(\cdot, F)$. The second assertion follows from Lemma 1.0 and the fact that pointwise convergence of $\left\langle d\left(\cdot, F_{n}\right)\right\rangle$ to $d(\cdot, F)$ forces Lim $F_{n}=F[3]$.

Example 1 of [3] shows that ${ }^{\tau} C$-convergence of $\left\langle F_{n}\right\rangle$ to $F$ does not ensure that $\left\langle d\left(\cdot, F_{n}\right)\right\rangle$ converges to $d(\cdot, F)$ pointwise. We now show that pointwise convergence of distance functions does not guarantee the convergence of the underlying sequence of sets in the ball topology.

EXAMPLE 2.1. Let $e_{n}$ be the real sequence whose $n$th term is 1 and whose other terms are all zero. Consider this subspace $X$ of $1_{\infty}$ :

$$
X=\{0\} \cup\left\{e_{n}: n \in Z^{+}\right\} \cup\left\{\left(\frac{n+1}{n}\right) e_{n} ; n \in Z^{+}\right\}
$$

Let $F=\left\{\left(\frac{n+1}{n}\right) e_{n}: n \in \mathrm{Z}^{+}\right\}$, and for each $n \in z^{+}$let $F_{n}=\left\{\left(\frac{j+1}{j}\right) e_{j}\right.$ : $j \leq n\} \cup\left\{e_{j}: j>n\right\}$. Since $\left\langle F_{n}\right\rangle$ actually $h_{d}$-converges to $F$, the sequence $\left\langle d\left(\cdot, F_{n}\right)\right\rangle$ converges pointwise to $d(\cdot, F)$. Although $F \in\left(B_{1}[0]^{c}\right)^{+}$, for each $n$ the set $F_{n}$ meets $B_{1}[0]$. Thus, $\left\langle F_{n}\right\rangle$ does not $\tau_{B}$-converge to $F$.

In view of the next lemma, it is reasonable to call the spaces $X$ presented in [3] spaces with nice closed balls. Not only do they include spaces in which closed and bounded sets are compact, but also spaces whose distance function is the zero-one metric.

LEMMA 2.2. Let $\langle X, d\rangle$ be a metric space. The following are equivalent:

(1) For each $p$ and $x$ in $X$, whenever $\left\langle x_{n}\right\rangle$ is a sequence in $X$ 
with no cluster point, then $d(p, x) \leq \lim$ inf $d\left(p, x_{n}\right)$;

(2) If $B$ is a noncompact closed ball in $X$, then $B=X$.

Proof. $(1) \rightarrow(2)$. Let $B_{\varepsilon}[p]$ be a noncompact closed ball in $X$. Let $\left\langle x_{n}\right\rangle$ be a sequence in the ball with no cluster point, and set $\alpha=\lim$ inf $d\left(p, x_{n}\right)$. By hypothesis, $X=B_{\alpha}[p]$. Since for each $n$ we have $d\left(p, x_{n}\right) \leq \varepsilon$, it follows that $\alpha \leq \varepsilon$. As a result, $X=B_{\varepsilon}[p]$.

$(2) \rightarrow(1)$. Fix $p \in X$ and let $\left\langle x_{n}\right\rangle$ be a sequence in $X$ with no cluster point. Condition (1) clearly holds if $\lim$ inf $d\left(p, x_{n}\right)=\infty$. There remains the possibility that $\lim$ inf $d\left(p, x_{n}{ }^{\prime}\right.$ is a finite number $\alpha$. Suppose for some $x \in X, d(p, x)>\alpha$. If $\varepsilon=\frac{1}{2}[\alpha+d(p, x)]$, then $B_{\varepsilon}[p]$ would contain infinitely many $x_{n}$. Thus, $B_{\varepsilon}[p]$ is neither compact nor is it all of $X$, in violation of (2).

THEOREM 2.3. Let $\langle X, d\rangle$ be a metric space. The following are equivalent:

(1) $X$ has nice closed balls!

(2) $\tau_{B}=\tau_{C}$ on $C L(X)$;

(3) ${ }^{2} C^{\text {-convergence of sequences in } C L(X)}$ is equivalent to the pointwise convergence of their distance functions.

Proof: (1) $\rightarrow(2)$. By the remarks made at the end of section 1 , $\tau_{C} \subset \tau_{B}$. The reverse inclusion is immediate from condition (1).

$(2) \rightarrow(3)$. By the second part of Lemma 2.0 , we need only show that if $\left\langle F_{n}\right\rangle$ is $\tau_{C}$-convergent to $F$, then $\left(d\left(\cdot, F_{n}\right)\right\rangle$ converges pointwise to $d(\cdot, F)$. But if (2) holds, this is immediate from the first part of Lemma 2.0 .

$(3) \rightarrow(1)$. Suppose $F, F_{1}, F_{2}, \cdots$ are nonempty closed sets in $X$

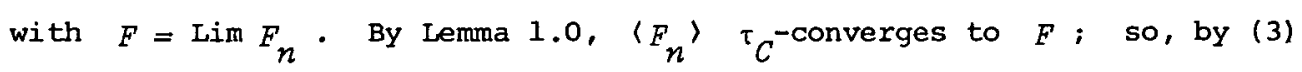
the associated sequence of distance functions is convergent. By Lemma 2.2 and Theorem 1 of [3], the space $X$ has nice closed balls. 
An immediate consequence of Theoren 2.3 is this: if $X$ has nice closed balls, then $\tau_{B}$-convergence of sequences in $C L(X)$ is equivalent to the pointwise convergence of their distance functions. The converse fails, a point that we shall return to following Theorem 2.7.

We now describe the precise place of the Hausdorff metric in the scheme of things. It is well-known and easy to show that the $h_{d}$-topology always contains the Fell topology, whereas Example 2.1 shows that it need not contain the ball topology. By virtue of the next lemma, the ball topology need not contain the $h_{d}$-topology either (see also Lemma 3.2 of [15]). The key to the proof is the following obvious fact: if $\left\langle F_{n}\right\rangle$ is an increasing sequence of nonempty closed sets in a metric space, then $\left\langle F_{n}\right\rangle$ converges to $\overline{\cup F_{n}}$ with respect to $\tau_{B}$.

LEMMA 2.4. Let $(X, d\rangle$ be a metric space. Suppose the ball topology on $C L(X)$ contains the Hausdorff metric topology. Then $X$ is totally bounded.

Proof. For any metric space $\langle X, d\rangle$ and for each $\varepsilon>0$, there exists a subset $A_{\varepsilon}$ of $X$ such that $X \subset S_{\varepsilon}\left[A_{\varepsilon}\right]$ and if $\{x, y\} \subset A_{\varepsilon}$, then $d(x, y) \geq \varepsilon$ (use zorn's Lemma). Suppose $X$ fails to be totally bounded; then an infinite $A_{\varepsilon}$ must exist. Let $A=\left\{x_{n}: n \in Z^{+}\right\}$be a countably infinite subset of an infinite $A_{\varepsilon}$. For each $n \in 2^{+}$let $F_{n}=\left\{x_{j}: j \leq n\right\}$. Since $A$ is closed, by our above remarks, $\left\langle F_{n}\right\rangle$ $\tau_{B}$-converges to $A$. Clearly, $\left\langle F_{n}\right\rangle$ does not converge to $A$ in Hausdorff distance.

We next strengthen Proposition 2.5 of [9].

THEOREM 2.5. Let $\langle X, d\rangle$ be a metric space. The following are equivalent: (1) $X$ is totally bounded; (2) Hausdorff metric convergence of sequences in $C L(X)$ is equivalent to the pointwise convergence of their distance functions. 
Proof. $(1) \rightarrow(2)$. By our remarks in the introduction, $h_{d}$-convergence is at least as strong as pointwise convergence of $\left\langle d\left(\cdot, F_{n}\right)\right\rangle$ do $d(\cdot, F)$, with no assumptions on $X$ whatsoever. Now suppose $X$ is totally bounded, and $\left\langle d\left(\cdot, F_{n}\right)\right\rangle$ converges pointwise to $d(\cdot, F)$. Let $A$ be a finite $\frac{\varepsilon}{3}$-dense subset of $X$, and choose $N$ so large that for each $n>N$ and each $x$ in $A$ we have $\left|d\left(x, F_{n}\right)-d(x, F)\right|<\frac{\varepsilon}{3}$. Since distance functions are Lipschitz continuous with Lipschitz constant one, it follows from the triangle inequality that

$$
\sup _{x \in X}\left|d\left(x, E_{n}\right)-d(x, F)\right| \leq \varepsilon \quad(n>N)
$$

Thus, $\left(d\left(\cdot, F_{n}\right)\right\rangle$ converges uniformly to $d(\cdot, F)$, and (2) follows

$(2) \rightarrow(1)$. If $\langle X, d\rangle$ is not totally bounded and $\left\langle F_{n}\right\rangle$ and $A$ are as described in the proof of Lemma 2.4 , then by Lemma $2.0\left\langle d\left(\cdot, F_{n}\right)\right\rangle$ converges pointwise to $d(\cdot, A)$.

Total boundedness of $X$ is not sufficient for the $h_{d}$-topology and the ball topology to agree on $C L(X)$. To spell out exactly what is needed in addition, we state another definition.

DEFINITION. Let $A$ and $B$ be disjoint subsets of a metric space $\langle X, d\rangle$. We say that $A$ and $B$ can be uniformly seporated if there exists $\varepsilon>0$ for which $S_{\varepsilon}[A] \cap S_{\varepsilon}[B]=\emptyset$.

We intend to show that the two topologies agree on $C L(X)$ if and only if $X$ is totally bounded, and it is possible to uniformly separate disjoint elements of $C L(X)$ whenever one of them is a closed ball. This last condition surely holds if $X$ has nice closed balls. It also holds if each pair of disjoint elements of $C L(X)$ can be uniformly separated. These are exactly the metric spaces on which continuous functions are always uniformly continuous [1]. In the literature such spaces are usually called UC spaces [19] or Atsuji spaces [4]. Euclidean $n$-space has nice closed balls, but fails to be a UC space. A UC space that fails to have nice closed balls is the "constellation" in $1_{\infty}:\{0\} \cup\left\{\frac{1}{j} e_{n}: j \in Z^{+}\right.$ and $\left.n \in \mathrm{Z}^{+}\right\}$. A space in which closed balls can be separated from closed 
sets (provided they are disjoint) which does not have nice closed balls and which is not a UC space is $(0,1)$, as a subspace of the line.

Our characterization will be obtained via the next lemma.

LEMMA 2.6. Let $\langle X, d\rangle$ be a totally bounded metric space. Suppose it is possible to uniformly separate disjoint elements of $C L(X)$ when one of them is a closed balz. Then the ball topology on $C L(X)$ is second countable.

Proof. For each $n \in Z^{+}$let $E_{n}$ be a finite subset of $X$ for which $X \subset S_{1 / n}\left[E_{n}\right]$. We claim that

$$
{ }_{\left(S_{1 / j}[x]\right)^{-}}^{\left[\left(B_{1 / j}[x]\right)^{c}\right]^{+}} \quad\left(j \in Z^{+} \text {and } x \in{ }_{n=1}^{\infty} E_{n}\right)^{\prime}
$$

is a (countable) subbase for the ball topology. Since $U E_{n}$ is dense in $X$, it is clear that if $S_{\delta}[p]$ is an arbitrary open ball in $X$ and $F \in\left(S_{\delta}[p]\right)^{-}$then there exists $x \in \cup E_{n}$ and $j \in Z^{+}$for which $E \in\left(S_{1 / j}[x]\right)^{-} \subset\left(S_{\delta}[p]\right)^{-}$. On the other hand, let $B$ be a closed ball in $X$ and suppose $A \in\left(B^{c}\right)^{+}$. By assumption, $A$ and $B$ can be uniformly separated; so, there exists $n \in Z^{+}$for which $A \cap S_{2 / n}[B]=\emptyset$. Choose $E \subset E_{n}$ for which $B \subset S_{1 / n}[E]$ and for each $x \in E$ we have $d(x, B)<1 / n$. It follows that $U\left\{B_{1 / n}[x]: x \in E\right\}$ contains $B$ and is contained in $S_{2 / n}[B]$. As a result,

$$
A \in \underset{x \in E}{n}\left[\left(B_{1 / n}[x]\right)^{C}\right]^{+} \subset\left(B^{c}\right)^{+}
$$

THEOREM 2.7. Let $(X, d)$ be a metric space. The following are equivalent: (1) The Hausdorff metric topology on $C L(X)$ equals the ball topology: (2) $X$ is totally bounded, and it is possible to uniformly separate disjoint elements of $C L(X)$ whenever one of them is a ball. 
Proof. $(1) \rightarrow(2)$. By Lemma 2.4, $X$ is totally bounded. If $A \in C L(X)$ and $B$ is a closed ball disjoint from $A$, then for some $\varepsilon>0$ the set $S_{\varepsilon}[A]$ must fail to meet $B$; otherwise; $\left\langle\overline{\left.S_{1 / n}[A]\right\rangle}\right.$ would be $h_{d}$-convergent, but not $\tau_{B}$-convergent, to $A$. We conclude that $S_{\varepsilon / 2}[A] \cap S_{\varepsilon / 2}[B]=\varnothing$.

$(2) \rightarrow(1)$. With no assumptions on $X$ each set $\left(S_{\varepsilon}[x]\right)^{-}$is $h_{d}$-open. Now suppose $A \in C L(X)$ fails to meet a closed ball $B$. Choose $\varepsilon>0$ for which $S_{\varepsilon}[A] \cap S_{\varepsilon}[B]=\emptyset$. It follows that if $h_{d}(A, F)<\varepsilon$, then $F \cap B=\emptyset$. As a result, $\left(B^{c}\right)^{+}$contains an $h_{d}$-neighborhood of each of its points. We conclude that the $h_{d}$-topology is at least as strong as the ball topology. To show that $\tau_{B}$ is at least as strong as the $h_{d}$-topology, we may procede sequentially by virtue of Lemma 2.6 . Let $\left(F_{n}\right\rangle$ be a sequence in $C L(X) \tau_{B}$-convergent to a closed nonempty set $F$. By Lemma 2.0, $\left\langle d\left(\cdot, F_{n}\right\rangle\right\rangle$ converges pointwise to $d(\cdot, F\rangle$. Since $X$ is totally bounded, Theorem 2.5 guarantees that $\left\langle E_{n}\right\rangle h_{d}$-converges to $F$.

It follows from Lema 2.0 and Theorem 2.5 that if $X$ is a space satisfying condition (2) of Theorem 2.7 , then $\tau_{B}$-convergence of sequences in $C L(X)$ is equivalent to the pointwise convergence of their distance functions. As we noted earlier, $(0,1)$ as a subspace of the line is such a space. It is natural to guess that the total boundedness of $X$ is irrelevant here, and to make this conjecture: for $\tau_{B}$-convergence of sequences in $C L(X)$ to be equivalent to the pointwise convergence of their distance functions, it is both necessary and sufficient that closed balls can be uniformly separated from closed sets in $X$. We now pursue this question.

LEMMA 2.8. Let $\langle X, d\rangle$ be a metric space. Suppose $\tau_{B}$-convergence of sequences in $C L(X)$ is equivalent to the pointwise convergence of their distance functions. Then it is possible to uniformly separate disjoint elements of $C L(X)$ whenever one of them is a closed balz. 
Proof: Suppose there exist $A \in C L(X)$ and a closed ball $B$ which are disjoint, yet cannot be uniformly separated. We can find sequences $\left\langle x_{n}\right\rangle$ in $A$ and $\left(y_{n}\right\rangle$ in $B$ for which $\lim _{n \rightarrow \infty} d\left(x_{n}, y_{n}\right)=0$. Neither $\left\langle x_{n}\right\rangle$ nor $\left\langle y_{n}\right\rangle$ can have a cluster point, for it would lie in $A \cap B$. As a result, for each $n \in z^{+}$the set $F_{n}=\left\{x_{1}, \ldots, x_{n}, y_{n+1}, y_{n+2}, \ldots\right\}$ is closed. If we set $F=\left\{x_{n}: n \in z^{+}\right\}$, then $\left\langle d\left(\cdot, F_{n}\right\rangle\right\rangle$ converges pointwise to $d(\cdot, F)$. However, although $F \in\left(B^{c}\right)^{+}$, for each $n$ we have $F_{n} k\left(B^{c}\right)^{+}$. Thus, $\left\langle F_{n}\right\rangle$ fails to converge to $F$ in the ball topology.

It is a great disappointment to this author that the converse of Lemma 2.8 fails.

EXAMPLE 2.9. In $1_{\infty}$ let $F=\left\{\frac{n+1}{n} e_{1}+\frac{1}{2} e_{n}: n=2,3,4, \ldots\right\}$. We take for our space $X$ this subspace of $1_{\infty}$ :

$$
X=\{0\} \cup F \cup\left\{e_{1}+e_{n}: n=2,3,4 \ldots\right\}
$$

Notice that if $x$ and $y$ are distinct points of $X$, then $d(x, y) \geq 1 / 2$; so, $X$ is actually a UC space. For each $n \in 2^{+}$let $F_{n}=F \cup\left\{e_{1}+e_{k}: k>n\right\}$. Each $F_{n}$ meets $B_{1}[0]$, whereas $F$ does not; so, $\left(F_{n}\right)$ fails to converge to $F$ in the ball topology. We show that for $x$ in $X, \lim _{n \rightarrow \infty} d\left(x, F_{n}\right)=d(x, F)$. Two cases are trivial: if $x=0$, then for each $n d\left(x, F_{n}\right)=d(x, F)=1$, and if $x \in F$, then for each $n d\left(x, F_{n}\right)=d(x, F)=0$. Now suppose that $x \in F^{c}$ is nonzero. For some $n \geq 2$, we have $x=e_{1}+e_{n}$. Evidently, for each $y \in F^{c}-\{x\}$ we have $d(x, y)=1$. On the other hand, $d(x, F)=d\left(e_{1}+e_{n}, \frac{n+1}{n} e_{1}\right.$ $+\frac{1}{2} e_{n}^{\prime}=1 / 2$. As a result, for this fixed $x$

$$
d\left(x, F_{k}\right)=\left\{\begin{array}{cc}
0 & \text { if } k<n \\
d(x, F) & \text { if } k \geq n
\end{array}\right.
$$

We conclude that $\lim _{n \rightarrow \infty} d\left(x, F_{n}\right)=d(x, F)$ in this last case, too. 
To finish this section, we include a new proof of a known result, and in the process, show that spaces with nice closed balls are complete.

THEOREM 2.10. Let $\langle X, d\rangle$ be a metric space. Then the Hausdorff metric topology on $C L(X)$ coincides with the Fell topology if and only if $X$ is compact.

Proof: If $X$ is compact, all of the notions of convergence we have considered coincide. Conversely, if the Hausdorff metric topology on $C L(X)$ coincides with the Fell topology, then by Theorem 2.3 and Theorem 2.5, the space has nice closed balls and is totally bounded. We show that the former condition implies that $X$ is complete. suppose $\left\langle x_{n}\right\rangle$ is a Cauchy sequence in $X$ with distinct terms without a cluster point. Then, $\alpha=\inf \left\{d\left(x_{1}, x_{n}\right): n>1\right\}$ is positive. Choose $N \in Z^{+}$such that whenever $n>m \geq N$ we have $d\left(x_{n}, x_{m}\right)<\alpha / 2$. It follows that $B_{\alpha / 2}\left[x_{n}\right]$ is a noncompact proper subset of $X$, a contradiction. We conclude that $\left\langle x_{n}\right\rangle$ has a cluster point; so, $X$ is complete. Since $X$ is also totally bounded, $X$ is compact.

3. Nearest points, furthest points and spaces with nice closed balls

By a multifunction $\Gamma$ from a metric space $X$ to a metric space $Y$, we mean a function from $X$ to $C L(Y)$. Now let $F$ be a nonempty closed set in a metric space $\langle X, d\rangle$. We say that $F$ is proximinal [7] if for each $x \in X$ there exists at least one point of $F$ nearest $x$. If $F$ is proximinal, we call the multifunction $\mathrm{r}_{F}: X \rightarrow C L(X)$ defined by

$$
\Gamma_{F}(x)=\{y \in F: \quad d(x, y)=d(x, F)\}
$$

the metric profection for $F$.

THEOREM 3.0. Let $\langle X, d\rangle$ be a metric space with nice closed balls, and let $F \in C L(X)$. Then:

(1) $F$ is proximinal, and for each $p \in X$ the set $\Gamma_{F}(p)$ is either compact or is all of $F$;

(2) The metric projection $\Gamma_{F}$ is upper semicontinuous in the sense of 
Kuratowski, therefore, whenever $V$ is open in $X$ then $\left\{x: \Gamma_{F}(x) \subset V\right.$ is open in $X$;

(3) If $p \in X$ has no furthest point in $F$, then sup $\{d(p, x): x \in F\}$ $=\sup \{d(p, x): x \in X\}$.

Proof: To prove (1), fix $p \in X$ and suppose all points of $F$ are not equidistant from $p$. Choose $x$ and $y$ in $F$ with $d(p, x)<d(p, y)$, and let $\alpha$ be between these two distances. Since $B_{\alpha}[p]$ is compact, the set of nearest points to $p$ in $B_{\alpha}[p] \cap F$ is a nonempty compact set. These are the points of $F$ nearest $p$. To prove (2), let $V$ be an open neighborhood of $\Gamma_{E}(p)$. If $\Gamma_{F}(p)=F$, then $\left\{x: \Gamma_{F}(x) \subset V\right\}=X$. Otherwise, $\Gamma_{F}(p)$ is a proper subset of $F$, and there exists $\alpha>0$ for which

$$
d(p, F)<\alpha<\sup \{d(p, x): x \in F\}
$$

Suppose $\left\{x: \Gamma_{F}(x) \subset V\right\}$ does not contain a neighbourhood of $p$. Then there exists a sequence $\left\langle x_{n}\right\rangle$ convergent to $p$ such that for each $n$ the set $\Gamma_{F}\left(x_{n}\right) \cap V^{c}$ is nonempty. Since $\lim _{n \rightarrow \infty} d\left(x_{n}, F\right)=d(p, F)<\alpha$, eventually $\Gamma_{F}\left(x_{n}\right) \cap V^{\mathcal{E}}$ lies within the compact set $B_{\alpha}[p]$. As a result, Ls $\left(\Gamma_{F}\left(x_{n}\right) \cap V^{\mathcal{E}}\right)$ is nonempty. Each point $q$ in this set lies in $F \cap V^{\mathcal{E}}$ and satisfies $d(p, q)=d(p, F)$, in violation of $\Gamma_{F}(p) \cap V^{c}=\emptyset$. To prove (3), suppose $p \in X$ has no furthest point in $F$ and $\beta=$ $\sup \{d(p, x): x \in F\}$ is finite. If $B_{\beta}[p]$ were compact, then $F$ would be compact, and a furthest point would exist, an impossibility. Thus $B_{\beta}[p]=X$. The case $B=\infty$ is trivial.

We intend to characterize spaces with nice closed balls in terms of nearest and furthest points. We need a simple fact for this purpose.

LEMMA 3.1. Let $\left\langle\alpha_{n}\right\rangle$ be a real sequence with distinct terms. Then $\left\langle\alpha_{n}\right\rangle$ has a monotone subsequence. 
Proof: A proof can be constructed through brute force, but the result follows most elegantly from Rarnsey's Theorem [17]: if the $n$ element subsets of $Z^{+}$are partitioned into $k$ classes $\left\{E_{1}, E_{2}, \ldots E_{k}\right\}$, then there exists an infinite subset $A$ of $Z^{+}$and $j \in\{1,2, \ldots, k\}$ such that each $n$-element subset of $A$ lies in $E_{j}$. We can get by here with $n=k=2:$ let $E_{1}=\left\{\{j, n\}: j<n\right.$ and $\left.\alpha_{j}<\alpha_{n}\right\}$ and let $E_{2}=\{\{j, n\}$ : $j<n$ and $\left.\alpha_{j}>\alpha_{n}\right\}$.

THEOREM 3.2. Let $\langle X, d\rangle$ be a metric space. The following are equivalent:

(1) X has nice closed balls;

(2) For each $F \in C L(X)$ and each $p \in X$ (i) the set of nearest points to $p$ in $F$ is either a nonempty compact set or is all of $F$, and (ii) if $p$ has no furthest point in $F$, then $\sup \{d(p, x): x \in F\}$ $=\sup \{d(p, x): x \in X\}$.

Proof: The implication (1) $\rightarrow(2)$ is immediate from Theorem 3.0. Conversely, suppose (1) fails. We can then find a closed ball $B_{\alpha}[p]$ contained properly in $X$ and a sequence of distinct terms $\left\langle x_{n}\right\rangle$ in the ball with no cluster point. We claim that $\left(d\left(p, x_{n}\right)\right)$ has a constant subsequence. If not, then by passing to a subsequence we can assurne $\left\langle d\left(p, x_{n}\right)\right\rangle$ has distinct terms. Invoking Lema 3.1 and once again passing to a subsequence, we can assure $\left\langle d\left(p, x_{n}\right)\right\rangle$ is monotone. If it were strictly decreasing, then the closed set $\left\{x_{n}: n \in Z^{+}\right\}$would have no point nearest $p$. If it were strictly increasing, then since $B_{\alpha}[p] \neq X$, we get

$$
\sup \left\{d\left(p, x_{n}\right): \quad n \in Z^{+}\right\} \leq \alpha<\sup \{d(p, x): x \in X\}
$$

In either case, condition (2) above is violated. We conclude that $\left\langle d\left(p, x_{n}\right)\right\rangle$ has a constant subsequence; in other words, there exists a closed noncompact subset $E$ of $B_{\alpha}[p]$ whose points are equidistant from $p$. Choose $q$ such that $d(p, q)>\alpha$, and form $F=E \cup\{q\}$. The set of nearest points to $p$ in $F$ is just $E$, a set which is neither compact 
nor all of $F$. Again, (2) is violated. We conclude that closed balls that are proper subsets of $X$ are compact.

EXAMPLE 3.3. The existence of unique nearest points in arbitrary elements of $C L(X)$ does not ensure that $X$ has nice closed balls. Let $X$ be this subspace of the plane: $\left\{\left(\frac{1}{n}, 0\right): n \in Z^{+}\right\} \cup\left\{\left(\frac{1}{3}, 1\right)\right\}$. It is easy to check that for each $p \in X$ and each $F \in C L(X)$ there exists a unique $x \in F$ satisfying $d(p, F)=d(p, x)$. However, the ball $B_{1}[(1,0)]$ is a noncompact proper subset of $X$.

\section{References}

[1] M. Atsuji, "Uniform continuity of continuous functions of metric spaces", Pacific J. Math. 8 (1958), 11-16.

[2] J.P. Aubin, Applied abstract analysis, (Wiley, New York, 1977).

[3] G. Beer, "On convergence of closed sets in a metric space and distance functions", Bulz. Australian Math. Soc. 31 (1985), 421-432.

[4] G. Beer, "Metric spaces on which continuous functions are uniformaly continuous and Hausdorff distance", Proc. Amer. Math. Soc. 95 (1985), 653-658.

[5] C. Berge, Topological spaces, (Oliver and Boyd, Edinburgh, 1963.).

[6] C. Castaing and M. Valadier, Convex conalysis and measurable multifunctions, (Springer-Verlag, Berlin, 1977).

[7] F. Deutsch and J. Lambert, "On continuity of metric projections", J. Approx. Theory 29 (1980), 116-131.

[8]. E. Effros, "Convergence of closed subsets in a topological space", Proc. Amer. Math. Soc. 16 (1965), 929-931.

[9] S. Francaviglia, A. Lechicki and S. Levi, "Quasiuniformization of hyperspaces and convergence of nets of semicontinuous multifunctions", J. Math. Anal. Appl. 112 (1985), 347-370. 
[10] W. Hildenbrand, Core and equilibria of a large economy, (Princeton University Press, Princeton, N.J. 1974).

[11] J. Kelley, General topology, (Van Nostrand, Princeton, N.J. 1955) .

[12] E. Klein and A. Thompson, Theory of correspondences, (Wiley, New York, 1984).

[13] K. Kuratowski, Topology, (Academic Press, New York, 1966).

[14] A. Lechicki and S. Levi, "Wijsman convergence in the hyperspace of a metric space", (to appear, Boll. Un. Mat. Ital.).

[15] E. Michael, "Topologies on spaces of subsets", Trans. Amer. Math. Soc. 71 (1951), 152-182.

[16] S. Mrowka, "On the convergence of nets of sets", Fund. Math. 45 (1958) 237-246.

[17] F. Ramsey, "On a problem of formal logic", Proc. London Math. Soc. 30 (1930), 264-286.

[18] G. Salinetti and R. Wets, "Weak convergence of probability measures revisited", (in preparation).

[19] W. Waterhouse, "On UC spaces", Amer. Math. Monthly 72 (1965), 634-635.

Department of Mathematics,

California State University, Los Angeles,

Los Angeles,

California 90032

United States of America. 